\title{
Point localisation in patients with unilateral brain damage
}

\author{
A TARTAGLIONE,* AL BENTON, $\dagger$ L COCITO*, G BINO,* E FAVALE* \\ From the Clinica Neurologica dell'Università, Genova, Italy* and the Department of Neurology, University \\ of Iowa, USA†
}

SUMmARY The ability to reproduce the position of points in a plane was examined by a copying test in a control group and in unilaterally brain damaged patients. The procedure was designed to minimise the influence of visual field defects and of spatial hemi-inattention on performance. Accuracy of localisation and direction of errors were studied in each half of the plane. Analysis showed a greater impairment of localisation ability in the patients with right hemisphere disease; however, the performance of both hemispheric groups was characterised by a reduction of accuracy in half of the plane contralateral to the side of the lesion. Both hemispheric groups showed an abnormal direction of errors in the left half of the plane, but the two groups presented a different pattern of errors.

Localisation of points in a bounded plane, under the condition of free gaze, has teen repeatedly investigated $^{1-8}$ in order to evaluate how brain damage affects the ability to orient in extrapersonal space. However, although the importance of different cortical $^{5-11}$ and subcortical ${ }^{12-14}$ structures has been recognised, the rôle of each hemisphere with regard to the relevant aspects of localisation has not been settled.

Data regarding the accuracy of performance on the side of the lesion appear quite contradictory even when the same testing procedure ${ }^{5}$ is applied. Some findings suggest that patients with lesions of the right hemisphere attain a very low level of accuracy, implying that the minor hemisphere plays a dominant rôle in this performance. ${ }^{5}$ Other findings, however, indicate that the two hemispheres are equally relevant to the task, since the lesion of either one affects localisation in the contralateral half of the space. ${ }^{46}$ Similarly the analysis of the data regarding the direction of mislocalisation within each half of space leads to unexpected results. It has been reported that both patients with left and right

Address for reprint requests: Dr A Tartaglione, Istituto di Clinica Neurologica dell'Università di Genova, via A. De Toni 5, 16132 Genova, Italy.

Accepted 13 July 1981 hemisphere lesions show a clear-cut tendency to shift the localisation of points towards the side of the lesion, suggesting the existence of contralateral neglect. $^{341516}$ However, the shift may be more prominent after lesion of the left hemisphere. ${ }^{4}$ This result seems to contradict clinical observations which relate unilateral neglect and distorted perception of spatial relationships to lesions of the right hemisphere. ${ }^{17}$ These contradictions might be explained in terms of the complexity of the task employed which often calls for the localisation of several points simultaneously. ${ }^{5}$ Undoubtedly in this case the difficulty of the performance is increased by the necessity to evaluate a large set of relative distances. Furthermore, some tasks call for a judgement of the configuration of points which is independent of the localising performance. Under this testing condition a derangement of performance after unilateral brain damage probably reflects a disturbance in complex spatial thinking, which is not necessarily involved in the localisation of single points. Finally the interaction of such factors as visual field defects or hemi-inattention might be responsible for the variability of the findings.

This study was designed to re-examine the relationships between hemispheric lesion and ability to localise points in a bounded plane. A very simple test was devised, to minimise the influence of the 
previously mentioned factors on the performance, and both the accuracy of localisation and the orientation of errors within the plane were analysed.

\section{Materials and methods}

\section{Subjects}

In a cooperative study of the Department of Neurology of the University of Iowa (USA) and of Genova (Italy), the test was given to 146 patients. Forty-eight control patients ( 24 from Iowa and 24 from Genova) who showed no history or evidence of brain disease, were drawn from a large sample which we have already reported on. ${ }^{18}$ Within each nationality subgroup 12 patients performed with the preferred hand and 12 used the non-preferred hand. Since a previous study of the control patients had shown that hand and nationality do not affect the performance, ${ }^{18}$ the four groups will be considered as a whole.

Fifty-three patients ( 22 from Iowa and 31 from Genova) had lesions confined to the left hemisphere as determined by the clinical findings, angiography and isotope brain scan. Forty-five patients (16 from Iowa and 29 from Genova) had lesions confined to the right hemisphere.

The mean ages of control, left hemisphere and right hemisphere groups were respectively 60 years $(\mathrm{SD}=9)$, 57 years $(\mathrm{SD}=11)$ and 56 years $(\mathrm{SD}=14)$. Differences of age among the three groups as well as between nationalities within the same group were not significant. All patients were right handed. Thirty-four patient in the left hemisphere group were clinically aphasic and nine showed a visual field defect (VFD) at the kinetic perimetry or the confrontation test. No patient in the right hemispheric group was aphasic and 14 showed a left VFD. The brain damaged groups were reasonably matched for severity of sensory and motor disturbances. Cerebrovascular disease was the most frequent diagnosis $(55 \%$ in the left hemisphere group; $56 \%$ in the right hemisphere group). Neoplasm was the next most frequent diagnosis $(36 \%$ and $40 \%$ respectively in the left and in the right hemisphere groups). In surgical cases, the test was given before operation.

\section{Testing procedure}

A modified version of the "copying test"5 was used, consisting of a series of 12 white stimulus cards $(26.5 \mathrm{~cm} \times 20 \mathrm{~cm})$, six with one cross and six with two crosses. The cards were consecutively presented, held vertically, at a comfortable distance from the patients, who had been instructed to reproduce the location of each cross as accurately as possible on a response sheet of the same size as the stimulus card. The brain diseased patients used the hand ipsilateral to the side of the lesion. No time limit was imposed. The examiner called the patient's attention to any crosses that he had neglected to copy. It has been found that some subjects, both among the older controls and brain damaged patients, show a tendency to reproduce the position of the cross in the response card in a reverse manner with respect to that of the test stimulus, for example, crosses near the lower edge of the stimulus sheet may be located close to upper border in the response card and so on. These subjects were discarded.

\section{Scoring technique}

Each response card was superimposed on a quadriruled mastercard, the side of each square being $5 \mathrm{~mm}$. The displacement was measured and recorded in terms of the number of squares separating the reproduction from the original. The accuracy of performance as well as the direction of errors were rated for each cross.

(A) Accuracy of performance The number of squares separating the centre of the copied cross from the position of the corresponding one in the testing stimulus was computed along each axis, yielding two measures, one for the horizontal deviation and the other for the vertical deviation from the original. The individual horizontal and vertical scores in the left and in the right half of the card were respectively summed in order to give a horizontal deviation score (HDS) and a vertical deviation score (VDS) for each half of the card. The sum of these scores for each patient constituted an individual half page error which was considered to be a measure of accuracy within each half of the plane. Adding the left and right half page errors gave an index of the patient's accuracy in the whole plane, which will be referred to as the overall error.

(B) Direction of errors The horizontal and vertical scores were also regarded as vector scores by adding a plus or minus sign according to the direction of the displacement. In the case of errors along the horizonta $\mathbb{R}$ axis "plus" indicates a displacement towards the right居 and "minus" a displacement towards the left. Similarly $\sigma$ for the vertical error, "plus" and "minus" indicate respectively an upward or downward displacement. The्ठ scores of the crosses located in the same half of the card were summed algebraically in order to give a horizontaf vector score (HVS) and a vertical vector score (VVS) for each subject. The analysis of the direction of errors was limited to the HVSs of each half of the card and to their sum which will be referred to as the overall horizontal vector.

\section{Results}

\section{ACCURACY OF PERFORMANCE}

Table 1 presents the distribution of the individual overall errors of the control and brain damaged groups. It is clear that the right hemisphere group did more poorly than the left group. Nineteen of 45 patients $(42 \%)$ with right hemisphere disease scored below the cut-off level of the normal group, defined as the poorest performance of the controls, while only seven of 53 patients $(13 \%)$ with left hemisphere damage performed below the cut-off.

The individual HDSs and VDSs were subjected to a mixed multifactorial analysis of variance with one between-subject factor (presence of lesion) and two within-subject factors (half page and component of error, that is HDS vs VDS). ${ }^{19}$ In order to control for the heterogeneity of variance, the analysis was carried out on log-transformed data and, given the 
Table 1 Distribution of individual error scores

\begin{tabular}{llll}
\hline & Controls & $\begin{array}{l}\text { Left } \\
\text { hemisphere }\end{array}$ & $\begin{array}{l}\text { Right } \\
\text { hemisphere }\end{array}$ \\
\hline $25-29$ & 2 & & \\
$30-34$ & 4 & & 1 \\
$35-39$ & 7 & 7 & 2 \\
$40-44$ & 8 & 8 & 1 \\
$45-49$ & 6 & 5 & 3 \\
$50-54$ & 4 & 6 & 3 \\
$55-59$ & 5 & 3 & 1 \\
$60-64$ & 4 & 3 & 2 \\
$65-69$ & 2 & 4 & 4 \\
$70-74$ & 2 & 6 & 4 \\
$75-79$ & 1 & 3 & 2 \\
$80-84$ & 3 & 1 & 3 \\
$85-89$ & & 1 & 3 \\
$90-94$ & & 2 & 3 \\
$95-99$ & & 2 & 3 \\
$100-104$ & & 1 & 2 \\
$105-109$ & & & 1 \\
$110-114$ & & & 2 \\
$115-119$ & & & 3 \\
$120-124$ & & 1 & $83 \cdot 31$ \\
$125-129$ & & $61 \cdot 83$ & $34 \cdot 64$ \\
130 and over & & $25 \cdot 20$ & \\
Mean & $51 \cdot 40$ & &
\end{tabular}

different number of patients in each group, an unweighted mean solution was applied. ${ }^{19}$

Table 2 presents the mean HDSs and VDSs within each half card of the three groups of patients,

Table 2 Mean horizontal (HDS) and vertical deviation scores $(V D S)$

\begin{tabular}{|c|c|c|c|c|}
\hline & \multicolumn{2}{|c|}{ Left half page } & \multicolumn{2}{|c|}{ Right half page } \\
\hline & $H D S$ & $V D S$ & $H D S$ & $V D S$ \\
\hline Controls & $13 \cdot 54$ & $11 \cdot 46$ & $12 \cdot 96$ & $13 \cdot 44$ \\
\hline Left hemisphere & $14 \cdot 85$ & 13.91 & $17 \cdot 06$ & 16.57 \\
\hline Right hemisphere & $23 \cdot 16$ & $20 \cdot 27$ & $17 \cdot 87$ & $22 \cdot 02$ \\
\hline
\end{tabular}

Table 3 Three factor univariate analysis of variance on log transformed individual deviation scores. Factors: $A=$ groups (control, left hemisphere, right hemisphere); $B=$ position in the page (left half, right half) $C=$ component of error (horizontal deviation score, vertical deviation score

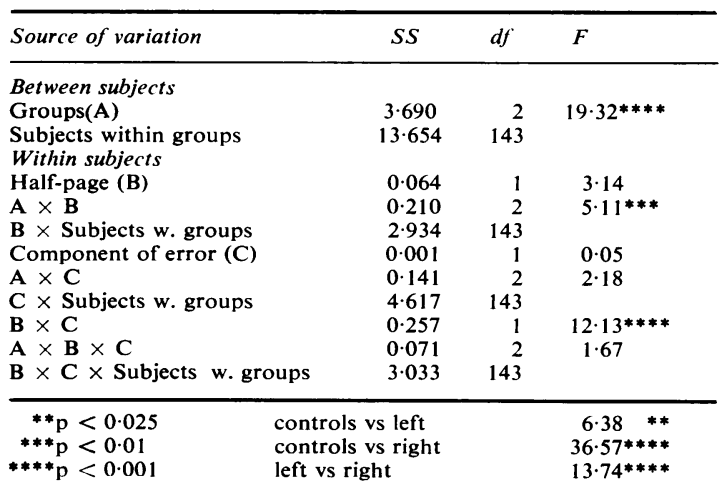

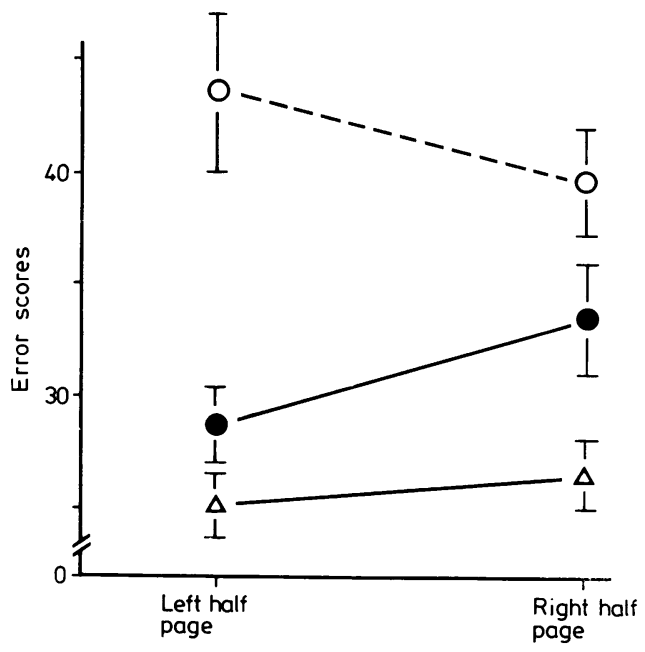

Fig 1 Mean half page errors of the three groups. Each error score corresponds to the average sum of individual $H D S$ and VDS for either half page and is expressed in conventional units. Triangles = controls; closed circles $=$ left hemisphere group: open circles = right hemisphere group.

and Table 3 is the summary of the analysis of variance. As will be seen, presence of lesion was characterised by an increase of error scores both in the left and in the right hemisphere groups. In addition, the right hemisphere group made more errors than the left hemisphere group.

The interaction between presence of lesion and half card is shown in fig 1 , where the mean half page errors for each group are reported. In both the control and the left hemisphere groups there were more errors in the right half of the card and the performance of the latter group was worse than that of controls $(F=8.7$; df: 1,$202 ; p<0.005)$. Conversely, in the right hemisphere group the difference between the left and right halves was reversed and the size of error was larger in the left half of the card $(F=4 \cdot 8 ; \mathrm{df}: 1,143: \mathrm{p}<0.05)$. The interaction between component of error (that is HDS vs VDS) and half card was statistically significant. The gradient of errors between the two halves of the card was determined more by a difference between the VDSs $(F=13.9$; df: 1,$286 ; p<0.001)$ than by a variation of the HDSs $(F=1 \cdot 5 ; \mathrm{df}: 1,286 ; n s)$.

Five $(36 \%)$ of the patients with right hemisphere lesion and visual field defects performed below the cut-off, while none of the nine patients in the left hemisphere group with visual field defects performed defectively. However, in a separate analysis, neither field defect $(\mathrm{F}=1 \cdot 3$; df:1,94; ns) nor its interactions with other variables reached the level of statistical significance. 

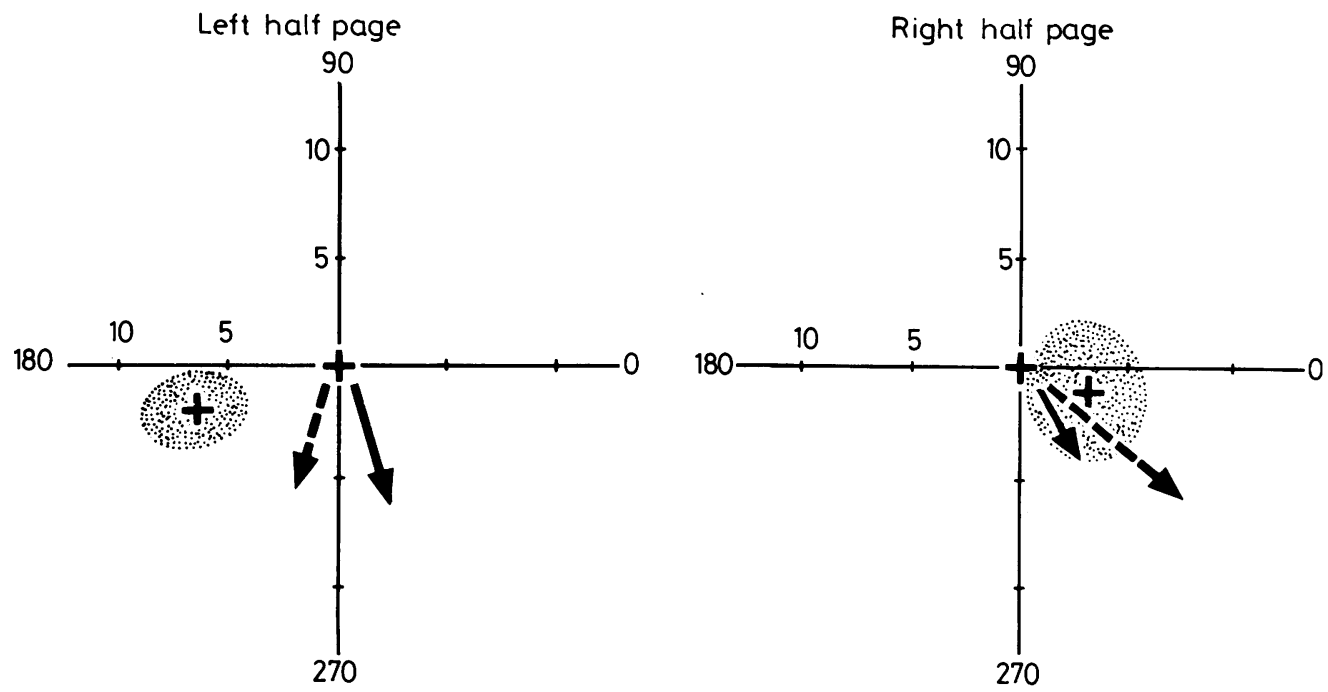

Fig 2 Polar plot of the mean directional error of three groups of patients in each half page, referred to the original position indicated by the central cross. Vectors relate to the performance of brain damaged patients and result from the mean HVS and VVS of each hemisphere group. Dashed vector = left hemisphere group; continuous vector $=$ right hemisphere group. The mean directional error of controls is indicated by the peripheral crosses inscribed in the $95 \%$ bivariate ellipse of confidence of mean.

\section{DIRECTION OF ERRORS}

Control as well as brain damaged patients showed a consistent tendency to deviate downwards, but the trend was enhanced in brain damaged patients (see fig 2). Table 4 presents the mean HVSs of the three groups for each half card. In view of the possibility that directional errors might be related to the accuracy of performance, the brain damaged patients was subdivided according to whether their level of accuracy was within normal limits or below. A factorial design of analysis of variance with a single control group ${ }^{19}$ was used, which permitted a comparison of the HVSs obtained by controls in each half of the card with the corresponding performances of the four groups of brain damaged patients, that is left and right hemisphere groups with normal or poor accuracy. The analysis was performed on individual HVSs after a log transform of their absolute values and an unweighted solution was applied. The summary of analysis of variance is shown in Table 5.

Table 4 Mean horizontal vector scores (HVS)

\begin{tabular}{|c|c|c|}
\hline$\therefore$ & Left half page & Right half page \\
\hline Controls & $-7 \cdot 21$ & +3.94 \\
\hline Left hemisphere $<$ cut-off & $\begin{array}{r}-1.63 \\
+1.14\end{array}$ & $\begin{array}{r}+5.96 \\
+10.00\end{array}$ \\
\hline Right hemisphere $\longleftrightarrow$ cut-off & $\begin{array}{r}-3.69 \\
+10.26\end{array}$ & $\begin{array}{r}+3.00 \\
+3.11\end{array}$ \\
\hline
\end{tabular}

The analysis indicates that presence of lesion, half $\overline{\mathrm{D}}$ card and the interaction between the two factors affect the direction of errors. The interaction indicates that the comparison between the HVSs of controls and those of brain damaged patients led to different outcomes in the two halves of the card. A difference between the performance of control and brain damaged groups was found only in the left half of the card $(F=12.5 ;$ df: 1,$246 ; p<0.001)$. Figure 2

Table 5 Three factor univariate analysis of variance on log transformed individual horizontal vector scores. Factors: $A$ = side of lesion (left hemisphere, right hemisphere); $B=$ accuracy (above or below the cut-off); $C=$ position in the page (left half, right half)

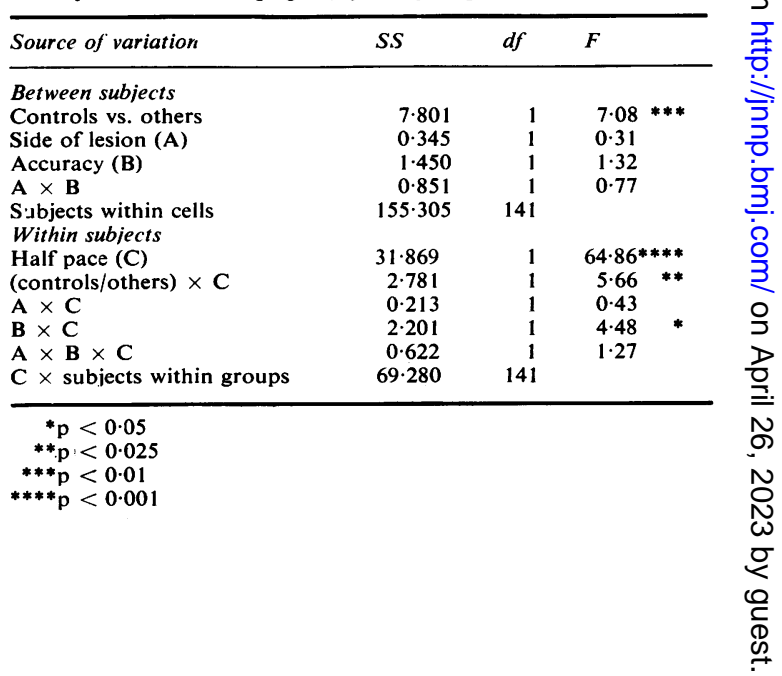


illustrates such a result in polar coordinates. The vectors resulting from the mean HVSs and VVSs of the control and brain damaged groups are presented, showing the mean value of the mislocalisation with respect to the position of the cross in the test card (central crosses). The performance of controls (peripheral crosses) is inscribed in the $95 \%$ bivariate ellipse of confidence of the mean. It is apparent that in the right half of the card all the patients showed
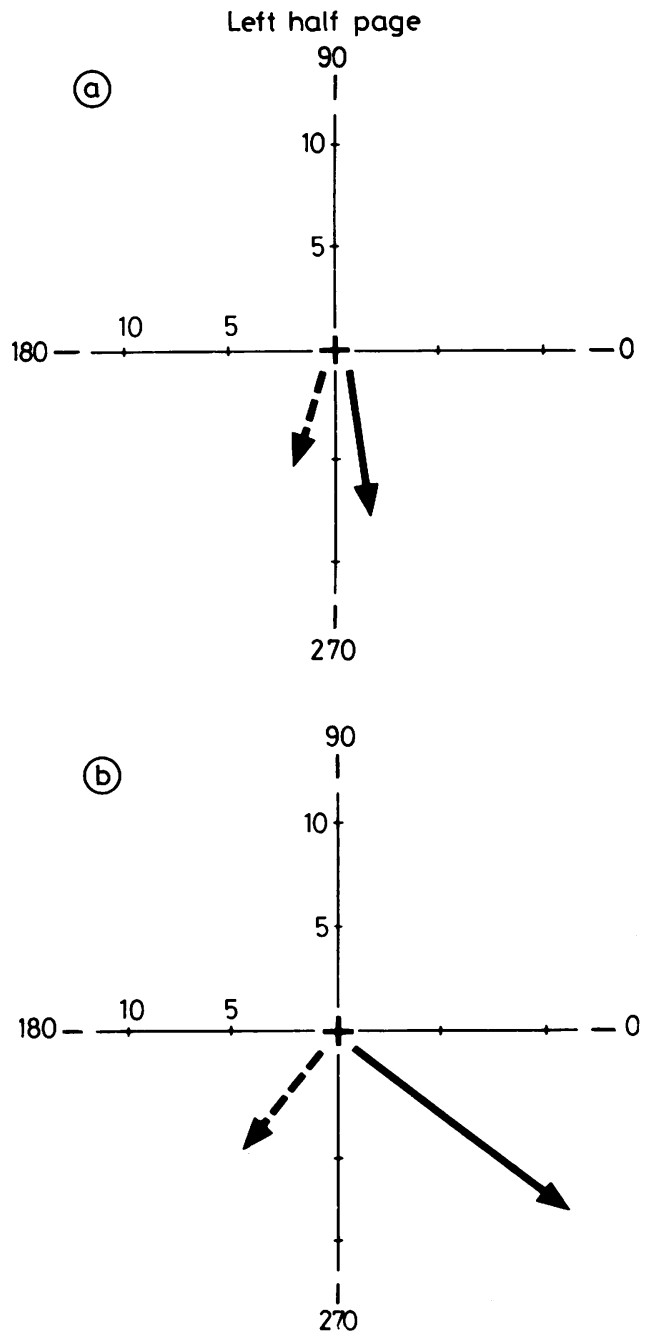

Fig 3 Polar plot of the mean directional errors (vectors) referred to the original position (central cross) produced in the left half page by brain damaged groups performing at different level of accuracy. $A=$ left hemisphere group; $B=$ right hemisphere group. Dashed line = normal accuracy group; continuous line $=$ low accuracy group. the normal rightward shift. In contrast, in the left half of the card the leftward shift of the control group was replaced by a quasi-zero mean HVS.

The interaction between accuracy and half card (see table 3 ) was statistically significant. The HVSs of patients who performed at a low accuracy level differed from those of other patients only in the left page $(F=4.5 ; \mathrm{df}: 1,246 ; \mathrm{p}<0.05)$.

Figure 3 presents the mean vectors obtained in the left half page by the two hemispheric groups at each level of accuracy. It is apparent that in the right hemisphere group a low level of accuracy was related to a large rightward shift, whereas normal accuracy was characterised by a leftward shift, as shown by the controls. The same patterns occurred in the left hemisphere patients. In this case the leftward and rightward shifts were very weak.

\section{Discussion}

The analysis of accuracy scores generated two main results to be considered. First, patients from each hemispheric group achieve a very low level of performance in the half space contralateral to the side of the lesion. This outcome indicates the importance of each hemisphere in the control of the crossed half of space. Furthermore, this pattern of errors is the only noticeable effect of a lesion of left hemisphere on the performance, since patients in this group do not differ from control as far as the performance in the half space ipsilateral to the lesion is considered.

Earlier results indicate that lesions of each hemisphere give rise to contralateral neglect. The latter can involve extrapersonal ${ }^{60-23}$ or intrapersonal ${ }^{24} 25$ space and it is particularly prominent in tasks making demands on capacity for visual search. ${ }^{22}$ Thus the present results might be related to a weaker control of contralateral half space, as one would expect in case of neglect. However, considering that none of our patients presented overt signs of hemi-inattention, it is more likely that our results were determined by a distorted perception of the spatial framework, as some authors have suggested.10

The second result has to do with the performances of the patients with disease of the right hemisphere who showed a low level of accuracy in both the halves of space. This supports the specific relevance of the right hemisphere in the evaluation of spatial relationship, as it has been demonstrated by a large body of evidences referring to point localisation 578 as well as to other performances. ${ }^{23} 2627$

The existence of a right hemisphere "dominance" for tasks such as point localisation is a controversial issue. Many researchers contend that processes 
through which objects are localised visually are organised in each hemisphere independently in respect to the crossed half space and are not related to the dominance of a single hemisphere. ${ }^{9-11} 2829$ Our results combine this point of view with the right hemisphere dominance hypothesis. 58 They suggest that localisation of points, as assessed by a copying performance, calls on two different abilities, each one depending on a different functional organisation of the hemisphere. One is related to the specific control of each half space and depends upon the integrity of both hemispheres, each one controlling the crossed half-space. The other is related to the ability to evaluate spatial relationships as distances or relative positions ${ }^{2326}$ and depends upon the integrity of the right hemisphere.

Visual field defects were not relevant to the performance either with respect to laalf page or overall errors. However, it is probable that our procedure, in which the examiner called the attention of the patients to skipped crosses, attenuated any effect of visual field defect.

With regard to the direction of error the performance of normal controls was mainly characterised by a large leftward shift in the left half of the card as opposed to a weaker rightward shift in the right half. ${ }^{18} 30$

From these results it is apparent that both hemispheric groups differ most sharply from the orienting behaviour of controls in the left half of the card. Specifically, left hemisphere patients show an average horizontal vector closely approaching zero, the individual performances being randomly directed and thus cancelling out in the algebraic sum. Conversely, the right hemisphere group shows an excessive rightward shift.

It has been suggested that directional deviations in locating points in the extrapersonal space might depend upon the existence of unilateral neglect. This would be responsible for a misplacement to occur away from the neglected side, that is, the half space contralateral to the lesion. ${ }^{41516}$ Our result failed to show a consistent pattern of errors except for a group of patients with right hemisphere lesion and this is in partial agreement with other data. ${ }^{3}$ In addition it is clear that orientation defects do not necessarily occur in the half space opposite to the lesion as it would be expected in case of unilateral neglect.

Our findings suggest that different mechanisms are probably involved in determining the direction of the shift in the two groups of brain damaged patients. In the right hemisphere group, the relationship between accuracy and directional shift suggests that the disruption of the same spatial mechanism may be responsible for both phenomena. Since the directional shift takes place in the same half space where the lowest level of accuracy is attained, that is the left half, it is conceivable that contralateral neglect plays a rôle in this case. In contrast, in the left hemisphere group, where the directional errors are not related to accuracy, other mechanisms have to be postulated, possibly praxic in nature, as some data would suggest. ${ }^{5}$

Further investigation along these lines is in progress to test these hypotheses. At present, it seems clear that simpler localisation tasks permit study of the main dimensions of such a spatial performance and increase the usefulness of such tasks as a diagnostic tool.

This investigation was supported by NATO Research Grant Nr 857 (Dr Tartaglione), CNR Grant $16 . \mathrm{CT} / 80.00439 .04 / 115.7743$ and by Grant NS OU 616 from the National Institute for Neurological and Communicative Disorders and Strokes (Dr Benton). We are indebted to Mrs Giuliana Cappati for help in testing the patients.

\section{References}

${ }^{1}$ Bender MB, Teuber HL. Spatial organisation of visual perception following injury to the brain. Arch Neurol Psychiatry 1947;58:721-39.

${ }^{2}$ Bender MB, Teuber HL. Spatial organisation of visual perception following injury to the brain. Arch Neurol Psychiatry 1948;59:39-62.

${ }^{3}$ Bisiach E, Capitani E, Colombo A, Spinnler H. Halving a horizontal segment: a study on hemisphere-damaged patients with cerebral focal lesions. Arch Suisses Neurol Neurochir Psychiatry 1976: 118:199-206.

${ }^{4}$ Capitani E, Pagani C, Spinnler H. Direction of spatial error in copying of visual stimuli: the relevance of focal brain damage. Arch Suisses Neurol Neurochir Psychiatry 1979;125:11-21.

${ }^{5}$ De Renzi E, Faglioni P. The relationship between visuo-spatial impairment and constructional apraxia. Cortex 1967;3:327-42.

${ }^{6}$ Faglioni P, Scotti G, Spinnler H. The performance of brain damaged patients in spatial localisation of visual and tactile stimuli. Brain 1971;94:443-54.

${ }^{7}$ Hannay HJ, Varney NR, Benton AL. Visual localisation in patients with unilateral brain disease. J Neurol Neurosurg Psychiatry 1976;39:307-13.

8 Warrington EK, Rabin P. Perceptual matching in patients with cerebral lesions. Neuropsychologia 1970;8:475-87.

9 Brain WR. Visual disorientation with special reference to lesions of the right cerebral hemisphere. Brain $1941 ; 64: 244-72$.

${ }^{10}$ Ratcliff G, Davies-Jones GAB. Defective visual localisation in focal brain wounds. Brain 1972; 95:49-60.

${ }^{11}$ Riddoch G. Visual disorientation in homonymous half fields. Brain 1935;58:376-482. 
12 Perenin MT, Jeannerod M. Residual vision in cortically blind hemifields. Neuropsychologia 1975;13:1-7.

${ }^{13}$ Perenin MT, Jeannerod M. Visual function within the hemianopic field following early cerebral hemidecortication in man. I Spatial localization. Neuropsychologia 1978;16:1-13.

${ }^{14}$ Weiskrantz L, Warrington EK, Sanders MD, Marshall J. Visual capacity in the hemianopic field following a restricted occipital ablation. Brain 1974;97:709-28.

${ }^{15}$ Benton AL, Levin NR, Van Allen MW. Geographic orientation in patients with unilateral cerebral disease. Neuropsychologia 1974;12:183-91.

${ }^{16}$ Colombo A, De Renzi E, Faglioni P. The occurrence of visual neglect in patients with unilateral cerebral disease. Cortex 1976;12:221-31.

${ }^{17}$ Hecaen H, Angelergues R. La cecité psychique. Paris: Masson, 1963.

${ }^{18}$ Tartaglione A, Benton AL, Raiteri U, Seneghini A, Cocito L, Favale E. Properties of localisation of points in a plane in normal subjects. J Gen Psychol $1981 ; 104: 133-43$.

19 Winer BJ. Statistical Principles in Experimental Design. New York: McGraw-Hill, 1971.

${ }^{20}$ Chain F, Leblanc M, Chedru F, Lhermitte F. Negligence visuelle dans les lesions posterieures de l'hemisphere gauche. Rev Neurol (Paris) 1979;135:105-26.

21 Costa LD, Vaughan HG, Horwitz M, Ritter W. Pattern of behavioral deficit associated with visual spatial neglect. Cortex 1969;5:242-63.

${ }^{22}$ De Renzi E, Faglioni P, Scotti G. Hemispheric contribution to exploration of space through the visual and tactile modality. Cortex 1970;6:191-203.

${ }^{23}$ De Renzi E, Faglioni P, Scotti G. Judgement of spatial orientation in patients with focal brain damage. J Neurol Neurosurg Psychiatry $1971 ; 34: 489-95$.

${ }^{24}$ Gainotti G, Tiacci C. The unilateral form of finger agnosia. An experimental study. Conf Neurol 1973; $35: 271-84$

${ }^{25}$ Denny-Brown D, Banker BQ. Amorphosynthesis from left parietal lesion. Arch Neurol Psychiatry 1954; 71:302-13.

${ }^{26}$ Benton AL, Hannay J, Varney NR. Visual perception of line direction in patients with unilateral brain disease. Neurology (Minneap) 1975;25:907-10.

${ }^{27}$ Benton AL. Disorders of spatial orientation. In : Vinken PJ, Bruyn GW, eds. Handbook of Clinical Neurology. Amsterdam: North Holland Publishing Co, 1969; vol 3:212-28.

${ }^{28}$ Cole M, Schutta HS, Warrington EK. Visual disorientation in homonymous half-fields. Neurology (Minneap) 1962;12:257-63.

29 Ettlinger G. Parietal cortex in visual orientation. In: Rose PC, ed. Physiological Aspects of Clinical Neurology. Oxford: Blackwell, 1977.

${ }^{30}$ Taylor MM. Effest of anchoring and distance perception on the reproduction of forms. Percept Mot Skills $1961 ; 12: 203-30$. 